\title{
New Method of Oil Reservoir Rock Heterogeneity Quantitative Estimation from X-ray MCT Data
}

\author{
Irena Viktorovna Yazynina ${ }^{1}$, Evgeny Vladimirovich Shelyago ${ }^{1, *} \mathbb{B}$, Andrey Andreevich Abrosimov ${ }^{1}$ \\ and Vladimir Stanislavovich Yakushev ${ }^{2}$ (D) \\ 1 Department of Oil Field Development and Operation, Gubkin Russian State University of Oil and \\ Gas (National Research University), 65 Leninsky Prospekt, 119991 Moscow, Russia; yazynina@mail.ru (I.V.Y.); \\ andreich.gis@gmail.com (A.A.A.) \\ 2 Department of Gas and Gas Condensate Field Development and Operation, Gubkin Russian State University \\ of Oil and Gas (National Research University), 65 Leninsky Prospekt, 119991 Moscow, Russia; \\ yakushev.v@gubkin.ru \\ * Correspondence: mail@fizikaplasta.ru
}

check for updates

Citation: Yazynina, I.V.; Shelyago, E.V.; Abrosimov, A.A.; Yakushev, V.S. New Method of Oil Reservoir Rock Heterogeneity Quantitative Estimation from X-ray MCT Data. Energies 2021, 14, 5103. https:// doi.org/10.3390/en14165103

Academic Editor: Kun Sang Lee

Received: 19 July 2021

Accepted: 16 August 2021

Published: 19 August 2021

Publisher's Note: MDPI stays neutral with regard to jurisdictional claims in published maps and institutional affiliations.

Copyright: (c) 2021 by the authors. Licensee MDPI, Basel, Switzerland. This article is an open access article distributed under the terms and conditions of the Creative Commons Attribution (CC BY) license (https:/ / creativecommons.org/licenses/by/ $4.0 /)$.

\begin{abstract}
This paper considers a new method for "pore scale" oil reservoir rock quantitative estimation. The method is based on core sample X-ray tomography data analysis and can be directly used to both classify rocks by heterogeneity and assess representativeness of the core material collection. The proposed heterogeneity criteria consider the heterogeneity of pore size and heterogeneity of pore arrangement in the sample void and can thus be related to the drainage effectiveness. The classification of rocks by heterogeneity at the pore scale is also proposed when choosing a reservoir engineering method and may help us to find formations that are similar at pore scale. We analyzed a set of reservoir rocks of different lithologies using the new method that considers only tomographic images and clearly distributes samples over the structure of their pore space.
\end{abstract}

Keywords: reservoir rock; heterogeneity; tomography

\section{Introduction}

Any oil or gas reservoir is heterogeneous, regardless of reservoir type. Heterogeneity may significantly distort the conclusions about oil and gas geological structure and lead to wrong solutions about hydrocarbons' extracting methods. Geo scientists and reservoir engineers often highlight reservoir heterogeneity as the reason for low oil and gas recovery [1]. Zonal and stratified inhomogeneity, strong variation in reservoir capacity and permeability lower the displacement ratio and sweep efficiency [2]. In laboratory practice, specialists discuss the sufficient core sample size and sample quantity for different experiments. On the one hand, the large number of large samples represents a better exploration opportunity; on the other hand, such studies are technically complex and may be characterized as expensive and time consuming. This problem is also close to the problem of scaling in hydrodynamic modeling [3]. Thus, we note that the problem of reservoir rock heterogeneity estimation has great practical significance.

Different specialists use the general term "heterogeneity" to describe one geological formation object, but they often put different meanings into the same concept. Geologists use heterogeneity to characterize macroscopic lithological composition, while reservoir engineers examine fluid flow and rock capacity properties. There is currently no single classification for the assessment of heterogeneity of geological objects. We note that quantitative characteristics of heterogeneity, which can be connected to displacement ratio and formation sweep efficiency, have great importance. Such characteristics may be used to analyze reservoir engineering systems and to explain deviations in field development parameters.

The main goal of this paper is to present a new method for quantifying the pore-scale heterogeneity of reservoir rock using X-ray tomography (MCT). 


\section{Existing Methods of Geological Object Heterogeneity Estimation}

In the broad sense, heterogeneity is the variation in lithological and physical properties of geological objects. Reservoir rock heterogeneity has been investigated by many outstanding scientists all over the world.

Geological heterogeneity is examined at various structural levels using the system approach. One of the first classifications of geological bodies by volumetric dimensions was proposed by Yu.A. Kosygin [4], where the whole set of bodies was divided into eight structural levels. The first level is for geological objects that are less than $1 \mathrm{~m}^{3}$ and the eighth level is for the Earth's crust and core. In petrophysics and petroleum geology, Russian scientists commonly use classifications by L.F. Dementev [5] and M.A. Tokarev [6], which include five and four heterogeneity levels, respectively.

It is important to understand the structural level of a geological body when studying heterogeneity. Geological heterogeneity is assessed qualitatively or quantitatively by using different statistical methods. Currently, there are several indicators that are used in petroleum geology.

In $[7,8]$, the coefficient of relative net-to-gross ratio is used to estimate the heterogeneity of the borehole section; the number of permeable intervals and the restraint coefficient (the fraction of the continuous formation thickness over the area) is used for the whole reservoir. G.M. Zoloeva suggests using the average vertical dissection of the object coefficient which characterizes the average frequency of layers with the alternation in different properties. Moreover, she suggests using the average vertical relative variability coefficient to analyze the heterogeneity of different geological formations [9]. V.D. Lysenko criteria (the square of the permeability variation coefficient), Dykstra-Parsons coefficient and Lorenz coefficient are used to estimate the reservoir permeability heterogeneity [10-12]. We should note that these indicators characterize the large-scale zonal or stratified heterogeneity and can be used to analyze the formation sweep efficiency during the reservoir development process.

A logical continuation of traditional methods is their integration [13]. This modern example describes the combination of mathematical statistics coefficients with Lorentz and Dykstra-Parsons coefficients to analyze well logging data.

Researchers have already recommended parameters for micro-scale heterogeneity characterization that are used in practice today [14]. These parameters are based on the analysis of grain-size composition, petrographic microsections, electronic microscopic data, etc. These methods are "planar»; they facilitate obtaining the rock image in a plane section. The existing parameters aimed to study the heterogeneity of mineral components, grains and crystals. However, scientists and reservoir engineers often point out that the oil and gas displacement process depends on micro-level pore structure, which always has three dimensions. In this regard, the current paper presents a new, simple way to measure and analyze 3D heterogeneity that forms the main difference between our method and existing ones.

\section{Materials and Methods}

It is important to note that the new heterogeneity criteria were introduced for the plug rock samples $(30 \times 30 \mathrm{~mm}$ cylinders). We used an X-Ray MCT method as one of the unique and actively developing tools for core research to obtain initial data about pore structure. MCT makes it possible to visualize voids (channels, pores, cracks) in the volume of the rock sample, to estimate the void sizes, and to trace their arrangement area $[15,16]$.

Currently there are several papers that address rock heterogeneity studies using MCT. In [17], the calculation of reservoir rock properties from MCT data illustrates that even a small plug sample is heterogeneous at the micro level and its areas are characterized by different porosity and permeability values which form the petrophysical trend. This trend may closely approach the whole formation trend, which can be obtained by summarizing trends from different rock samples [18].

D.V. Korost [19] developed the classification system for the quality assessment of reservoir rocks which is based on void structure analysis. The system includes visual 
analysis of 3D models obtained via MCT and does not provide quantitative results. The goal of this paper is to obtain the numerical characteristics of porous media heterogeneity, which can be used to compare different reservoir rocks, analyze core collection representativeness and analyze developing actions to achieve better oil recovery.

We suggest characterizing the pore space by means of two coefficients: the first one considers pore size heterogeneity while the second one considers the heterogeneity of pores' arrangement in the volume of a whole sample.

The pore size heterogeneity coefficient was taken by analogy with grain-size composition analysis [14]. In lithology and soil science, specialists use the $\mathrm{Cu}$ coefficient (uniformity coefficient), which characterizes grain size heterogeneity. A cumulative grain sizes distribution curve is plotted and analyzed to obtain the following values:

$d_{10}$ - the effective grain diameter ( $10 \%$ of grains in the rock are smaller than this size); $d_{60}$ - the control grain diameter ( $60 \%$ of grains in the rock are smaller than this size).

The heterogeneity coefficient $C_{h}$ is calculated as:

$$
C_{h}=\frac{d_{60}}{d_{10}}
$$

The deviation of $C_{h}$ from 1 increases with grain size heterogeneity growth. Because $\mathrm{MCT}$ enables researchers to obtain the pore size distribution, the approach described above was applied with a single change: we analyzed not grain size but pore size distribution. The coefficient similar to $C_{h}$ was named as the «pore size heterogeneity coefficient» $K_{h}(d)$. As the subject of our analysis is pore size distribution, the new coefficient is universal and can be calculated for both clastic and carbonate rocks.

The next step is to calculate the variation coefficient for porosity distributions to obtain the «pore arrangement heterogeneity coefficient» $K_{h}(V)$. In this case, high heterogeneity means that the pores are grouped into clusters inside the sample.

MCT is a 3D method to study objects that allows for layer-by-layer scanning. Each section of a rock sample can be scanned and its porosity value can be calculated. Subsequently, porosity distribution along the sample height can be plotted and analyzed (Figure 1). Similar distributions were obtained for all core samples involved in this study.
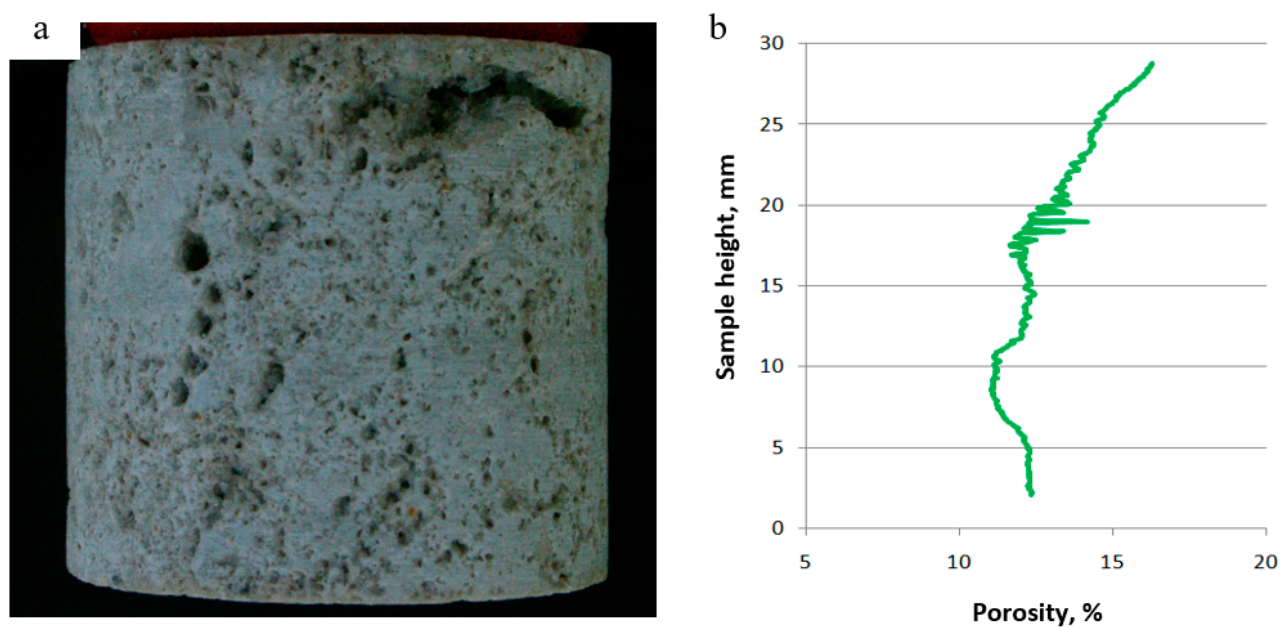

Figure 1. Petrophysical cylinder $(30 \times 30 \mathrm{~mm})($ a) and its porosity distribution in tomographic sections along the vertical axis of the sample (b).

If we have a one-directional value of the pore size, we can use the common «variation coefficient» formula to calculate the «pore arrangement heterogeneity coefficient»:

$$
W=\frac{\sigma}{\bar{x}} \cdot 100 \%
$$


$\sigma$ is the mean square deviation of the porosity coefficient that characterizes a parameter's dispersion near its mean value, and $\bar{x}$ is the mean value of porosity. In the future, we intend to refine the assessment of heterogeneity by analyzing different directions within each core sample.

The final step is to analyze the paired values of $K_{h}(d)$ and $K_{h}(V)$.

\section{Results and Discussion}

The paired values of heterogeneity coefficients $K_{h}(d)$ and $K_{h}(V)$ were calculated and plotted for the rock samples of eight different Russian oil formations (Figure 2).

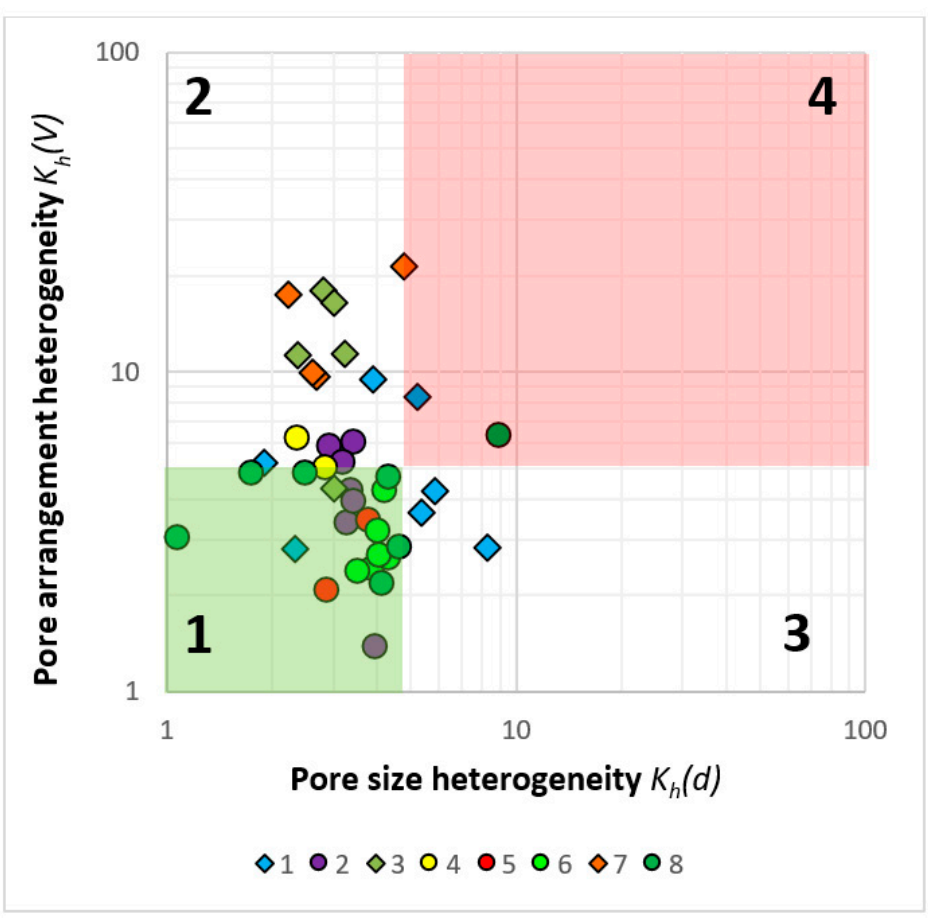

Figure 2. Heterogeneity coefficients for 8 groups of rock samples (8 different formations) according to MCT data analysis. Round markers: sandstones; diamond-shaped: carbonate. All values are calculated for $30 \times 30 \mathrm{~mm}$ cylinders.

Figure 3 presents the typical 3D pore-scale models (the model number corresponds to the legend number in Figure 2). We can visually distinguish between pore structures and, importantly, assign these differences in the form of specific values.

The plot area of Figure 2 was conventionally divided into four areas or classes. The class boundaries (five scale divisions along the $\mathrm{X}$ and $\mathrm{Y}$ axes) were subjectively chosen by analogy with the grain-size analysis, where reservoir rock was considered heterogeneous if $C_{h}$ was more than 5\%. These boundaries should be adjusted with the accumulation of actual data.

We can draw several conclusions about pore structure and its effect on oil recovery by the location of the heterogeneity values on the area-plot.

If reservoir rock heterogeneity is low, the sample will belong to Area 1. Therefore, uniform-size pores are regularly distributed in the sample's volume and, in this case, oil displacement cannot be complicated by features of the porous media structure.

If reservoir rock heterogeneity value belongs to Area 2, uniform-size pores are irregularly distributed in the sample's volume, i.e., these pores are located as separate groups (clusters). Oil displacement efficiency is strongly dependent on the interconnection between these groups resulting from small cracks and the spontaneous imbibition (or, for gas, the diffusion) ability of the displacing agent. Enhanced oil recovery can be achieved by stimulation of the spontaneous mass transfer. Changing the displacing agent properties is 
currently a realistic option. A more complex option is the initiation of rock cracking as it happens, for example, in the case of thermogas treatment [20].

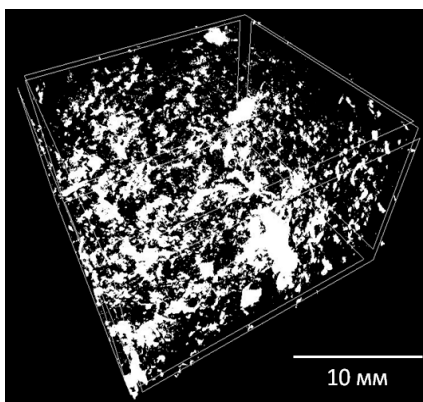

1-Pore type carbonate

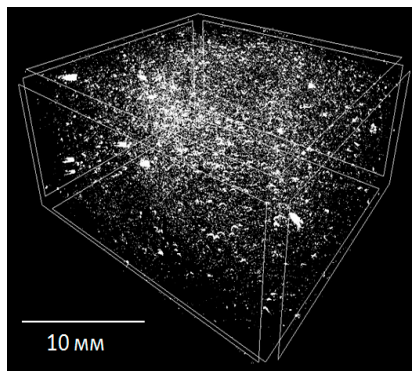

4-Sandstone

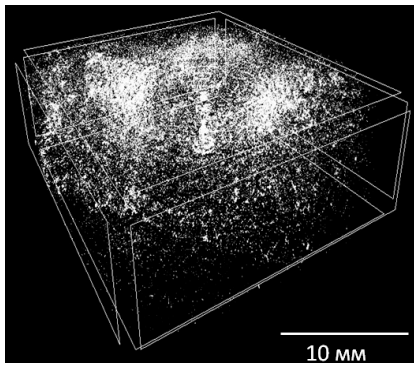

7-Crack-pore type carbonate
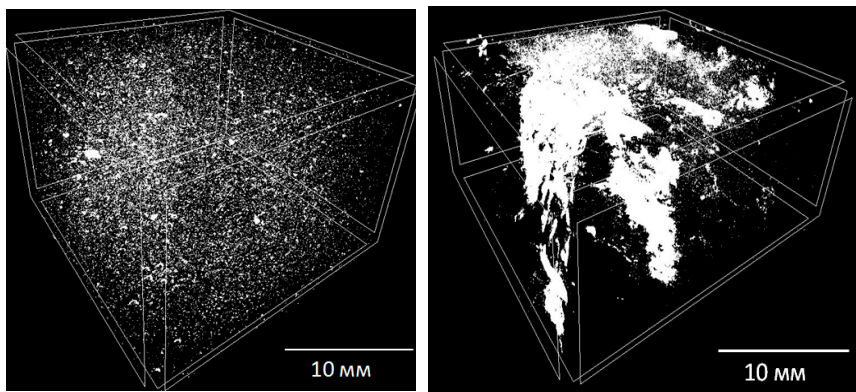

2-Sandstone with clay clusters

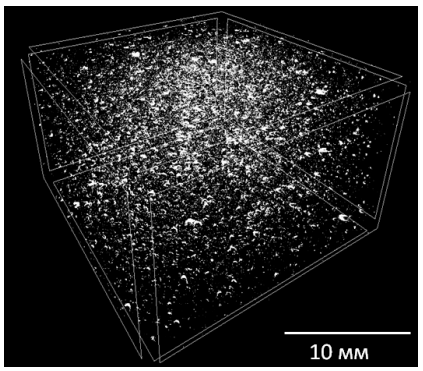

5-Sandstone

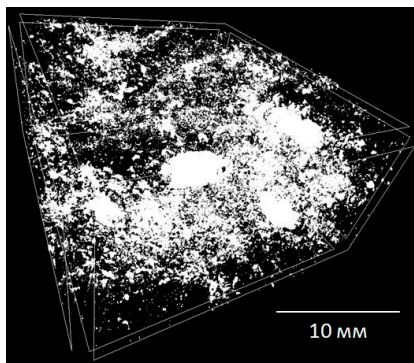

8-Sandstone with carbonate cement
3-Crack-pore type carbonate

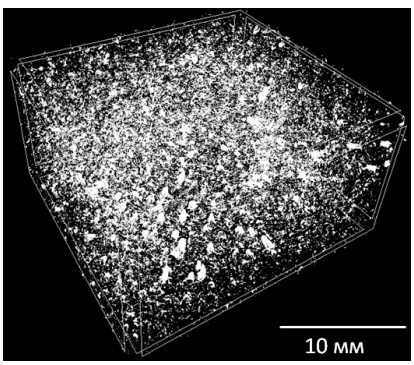

6-Sandstone

Figure 3. 3D pore-scale models for different formations. Samples are $30 \times 30 \mathrm{~mm}$ cylinders. MCT resolution is $10 \mu \mathrm{m}$ by Bruker SkyScan 1172.

A reservoir rock heterogeneity value belonging to Area 3 would mean that pores are regularly distributed in a sample's volume, but there is high heterogeneity in pore size. Residual oil would be trapped during the displacement process due to capillary effects. Additional oil recovery can be achieved by closing already-drained pores, interfacial tension and oil-water viscosity ratio change. The enhanced oil recovery can be achieved by regulation of capillary effects or closing of short flow trajectories as it happens, for example, in case of the polymer flooding [21].

If pore size and pore arrangement heterogeneity coefficients are high, the rock would belong to Area 4. Oil displacement efficiency would be minimal due to the high heterogeneity of the porous media structure.

The investigated rock samples are distributed in the proposed areas (classes) as follows: Crack-pore type carbonate (No. 3 and No. 7) and some sandstone samples with clay inclusions (No. 2) belong to Area 2, indicating that pores are located as clusters. A low permeable carbonate matrix or presence of clay interlayers is an obstacle between these clusters. Pore type carbonate (No. 1) is heterogeneous, with both types of heterogeneity presented in the pore structure; pore sizes vary over a wide range of values and can be 
grouped into separate clusters. Sandstone samples with carbonate cement (No. 8) have a wide variation in heterogeneity coefficients; values are located near Area 1 borders and both types of heterogeneity appear.

Additional information that can be obtained from the described heterogeneity analysis includes the representativeness of the core material collection in terms of scale. Estimation of the petrophysical properties and displacement ratio is based on the analysis of laboratory data that are measured for cylinder-shaped core samples (in Russia this is $30 \times 30 \mathrm{~mm}$; in other countries the size may differ) or for full-size core samples. The transfer to a large sample study happens due to the low reproducibility of experiments with small plugs and their low representativeness. Clearly, we obtain more objective information regarding actual formation properties when we measure large collections of big samples. However, there are technical, time and financial constraints that lead researchers to look for a "golden mean". In this paper, we propose the heterogeneity estimation method to determine this "golden mean". In fact, this method provides the answer to the following questions: how similar are the selected samples from the position of porous media structure heterogeneity, and must we use full-size samples instead of common plugs in laboratory experiments to obtain proper results?

It is necessary to analyze the standard deviation of heterogeneity coefficients for the current collection (Figures 3 and 4).

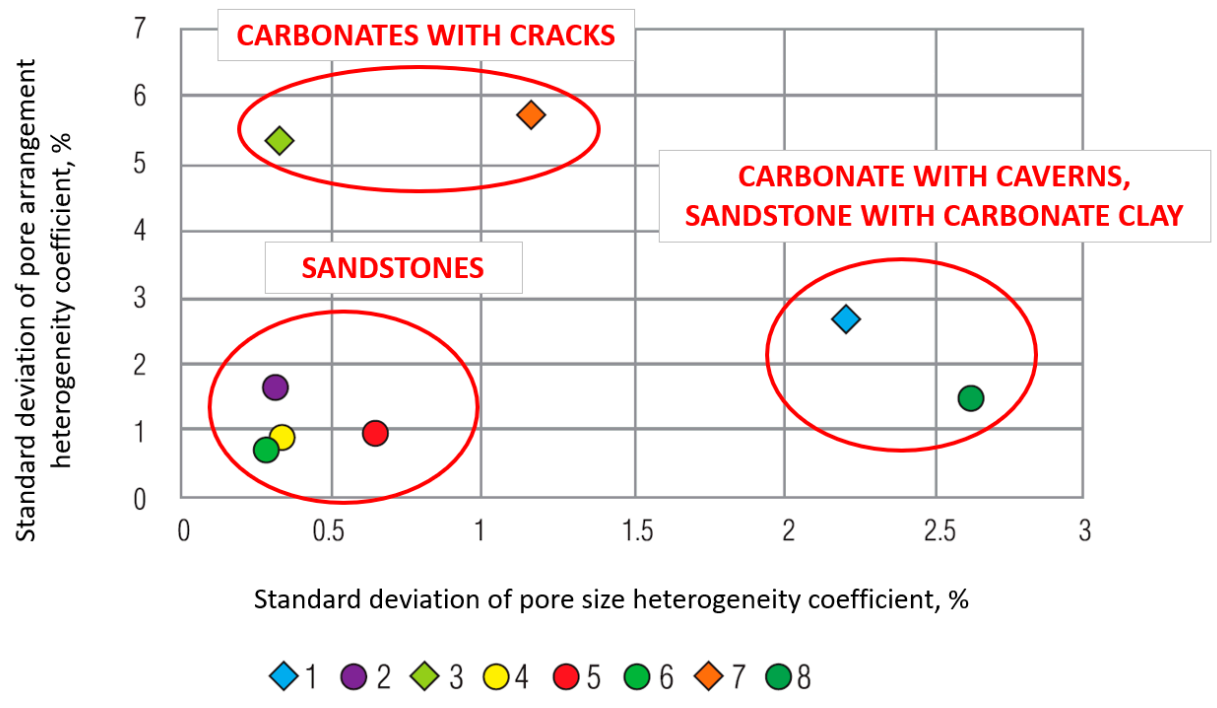

Figure 4. Standard deviation of heterogeneity coefficients indicates strong differentiation of tomographic images.

The standard deviation is low for samples that have similar porous media structures that may be heterogeneous but similar. For example, if we analyze the flow experiments that are carried out with such samples, we can compare the results for different plugs (or plug columns) and assume that the observed effects are unrelated to pore structure differences. If the standard deviation of heterogeneity coefficients for the current collection is high, this means that the selected sample size is not suitable for the purpose of comparison between different samples. The samples may have similar mineral composition, porosity, permeability, etc., but very different pore structures. In this case, we must increase the sample size to enhance the representativeness and obtain the ability to compare experimental results.

The analysis revealed that the carbonate samples (No. 1,3,7), as well as the sandstone samples with carbonate cement (No. 8), had strong standard deviations for heterogeneity coefficients. Therefore, we recommend studying the enlarged samples to ensure the conditions for comparing the experimental data with the indicated reservoirs are fulfilled. This result may be deemed predictable for carbonate rocks (although their heterogeneity is 
expressed as specific values after the analysis application) but, for the sandstone samples, this result could not be predicted at the early research stage.

In Russia, the formal transition to full-size core research is governed by Government Standard GOST 26450.0-85 (Mineral rocks: general requirements for selection and preparation of samples for reservoir properties measurement). In this standard, it is stated that full-size samples (equivalent to well-bore diameter) must be used if a rock includes the following elements: lithological characteristics that differ from the bulk sample volume; clear textural features (interlayers, plates etc.); or voids over $2 \mathrm{~mm}$ (caverns, cracks). The only quantitative indication is the presence of voids larger than $2 \mathrm{~mm}$. MCT heterogeneity analysis can be used for objective argumentation when choosing rock sample sizes in laboratory practice. The samples for the current job were previously cut by the petrophysical laboratory of an oil company as $30 \times 30 \mathrm{~mm}$ cylinders without regard to lithology, large voids, inclusions, etc.

We conclude that the proposed method of quantitative heterogeneity estimations from MCT data can be applied at any scale of tomographic data. This method is intended to supplement the existing rock classifications by heterogeneity. It can be used to analyze representativeness of the core material collection, reproducibility of the experimental data and to understand reasons for low oil displacement ratios. The method is easy and we hope to see it in wider use.

\section{Conclusions}

1. A new MCT-based numerical method can be used for quantitative estimation of the reservoir rock porous media heterogeneity. Two new coefficients (pore size heterogeneity coefficient and pore arrangement heterogeneity coefficient) are used for different lithological rock types. The use of new structure-dependent coefficients makes it possible to differentiate rock directly from tomographic images, which is novel for rock heterogeneity research.

2. Different reservoir rocks can be divided into classes depending on heterogeneity type (pore size and pore arrangement) and its value. The oil displacement ratio is reduced due to different features of the pore structure. These reasons are described for each class in this paper.

3. A new method for rock sample representativeness analysis is proposed thatshows the similarity of different samples in terms of porous media heterogeneity. The rock samples may have the same basic lithological description, close values of porosity and permeability but very different pore configurations, which significantly affect the results of laboratory measurements and eliminate the possibility of comparison. This method can also be used to approve the necessity of using enlarged samples in laboratory measurements.

Author Contributions: Conceptualization and methodology: I.V.Y., E.V.S.; software and formal analysis, visualization: A.A.A.; review and editing: V.S.Y. All authors have read and agreed to the published version of the manuscript.

Funding: This research received no external funding.

Institutional Review Board Statement: Not applicable.

Informed Consent Statement: Not applicable.

Data Availability Statement: Not applicable.

Conflicts of Interest: The authors declare no conflict of interest.

\section{References}

1. Muggeridge, A.; Cockin, A.; Webb, K.; Frampton, H.; Collins, I.; Moulds, T.; Salino, P. Recovery rates, enhanced oil recovery and technological limits. Philos. Trans. Ser. A Math. Phys. Eng. Sci. 2014, 372. [CrossRef]

2. Weber, K.J. How heterogeneity affects oil recovery. In Reservoir Characterization; Lake, B.L.W., Carroll, H.B., Eds.; Academy Press: Orlando, FL, USA, 1986. 
3. Sciencedirect. Reservoir Heterogeneity. Available online: https://www.sciencedirect.com/topics/engineering/reservoirheterogeneity (accessed on 13 July 2021).

4. Kosygin, Y.A. Fundamentals of Tectonics; Nedra: Moscow, Russia, 1974. (In Russian)

5. Dementev, L.F. Mathematical Methods and Computers in Oil and Gas Geology; Nedra: Moscow, Russia, 1983. (In Russian)

6. Tokarev, M.A. Integrated Geological and Technical Control over Current Oil Recovery in Water-Oil Displacement Process; Nedra: Moscow, Russia, 1990. (In Russian)

7. Semin, E.I. Different ways of studying geological heterogeneity of oil reservoirs. Trudi VNII 1962, 34, 3-43. (In Russian)

8. Stasenkov, V.V.; Klimushkin, I.M.; Breev, V.A. Oil Reservoir Geological Heterogeneity Research Methods; Nedra: Moscow, Russia, 1972. (In Russian)

9. Zoloeva, G.M. Estimation of Heterogeneity and Oil Recovery Forecast by Logging; Nedra: Moscow, Russia, 1995. (In Russian)

10. Lysenko, V.D.; Muharsky, E.D.; Hanzin, R.G. About oil reservoir heterogeneity. Trudi TatNII 1964, 6, 243-252. (In Russian)

11. Tiab, D.; Donaldson, E.C. Petrophysics: Theory and Practice of Measuring Reservoir Rock and Fluid Transport Properties, 4th ed.; Gulf Professional Publishing: Oxford, UK, 2015.

12. Lake, L.; Jensen, J.L. A Review of Heterogeneity Measures Used in Reservoir Characterization. IN SITU 1991, 15, 409-439.

13. Fitch, P.J.R.; Lovell, M.A.; Davies, S.J.; Pritchard, T.; Harvey, P.K. An integrated and quantitative approach to petrophysical heterogeneity. Mar. Pet. Geol. 2015, 63, 82-96. [CrossRef]

14. GOST-25100-2020. Soils. Classification. In Government Standard. Available online: https://docs.cntd.ru/document/1200174302 (accessed on 13 July 2021).

15. Abrosimov, A.A. X-ray tomography application in reservoir rock flow and capacity properties estimation. Gubkin Univ. Proc. 2015, 4/281, 5-15. (In Russian)

16. Blunt, M.J.; Bijeljic, B.; Dong, H.; Gharbi, O.; Iglauer, S.; Mostaghimi, P.; Paluszny, A.; Pentland, C. Pore-scale imaging and modelling. Adv. Water Resour. 2013, 51, 197-216. [CrossRef]

17. Yazynina, I.V.; Shelyago, E.V.; Abrosimov, A.A.; Veremko, N.A.; Grachev, N.E.; Senin, D.S. Novel approach to core sample MCT research for practical petrophysics problems solution. Oil Ind. 2017, 1, 19-23. (In Russian)

18. Yazynina, I.V.; Shelyago, E.V.; Abrosimov, A.A.; Veremko, N.A.; Grachev, E.A.; Bikulov, D.A. Testing a new approach to petrophysical trend determination from X-Ray tomography. Oil Ind. 2017, 2, 36-40. (In Russian)

19. Korost, D.V. MCT usage for clastic rock research. Oil gas Geol. 2010, 2, 36-42. (In Russian)

20. Kokorev, V.I.; Darischev, V.I.; Ahmadeyshin, I.A.; Schekoldin, K.A.; Bokserman, A.A. The impact of thermogas technologies on the Bazhenov Formation studies results. In Proceedings of the SPE Arctic and Extreme Environments Technical Conference and Exhibition, Moscow, Russia, 15-17 October 2013.

21. Zhong, H.; Zhang, W.; Fu, J.; Lu, J.; Yin, H. The Performance of Polymer Flooding in Heterogeneous Type II Reservoirs-An Experimental and Field Investigation. Energies 2017, 10, 454. [CrossRef] 